УДК 517.9

\title{
A study on fractional order theory in thermoelastic half-space under thermal loading
}

\author{
I.A. Abbas ${ }^{1,2}$ \\ ${ }^{1}$ Department of Mathematics, Faculty of Science, Sohag University, Sohag, 82524, Egypt \\ ${ }^{2}$ Nonlinear Analysis and Applied Mathematics Research Group, Department of Mathematics, \\ King Abdulaziz University, Jeddah, 21589, Saudi Arabia
}

In this study, the effect of fractional order derivative on a two-dimensional problem due to thermal shock with weak, normal and strong conductivity is established. The governing equations are taken in the context of Green and Naghdi of type III model (GNIII model) under fractional order derivative. Based on the Laplace and exponential Fourier transformations with eigenvalues approach, the analytical solutions has been obtained. For weak, normal and strong conductivity, the numerical computations for copper-like medium have been done and the results are shown numerically. The graphical results indicate that the effect of fractional order parameter has a major role on all physical quantities involved in the problem.

Keywords: eigenvalue approach, Green and Naghdi of type III model, fractional order generalized thermoelasticity

DOI $10.24411 / 1683-805 X-2018-11008$

\section{Дробное исчисление и его применение для описания термоупругого полупространства в условиях тепловой нагрузки}

\author{
I.A. Abbas ${ }^{1,2}$ \\ ${ }^{1}$ Сохагский университет, Сохаг, 82524, Египет \\ 2 Университет короля Абдулазиза, Джидда, 21589, Саудовская Аравия
}

\begin{abstract}
В работе показано влияние производной дробного порядка на решение двумерной задачи о тепловой нагрузке с низкой, нормальной и высокой проводимостью. Определяющие соотношения выбраны в рамках модели Грина-Нагди типа III (GNIII) для производной дробного порядка. На основе преобразования Лапласа и экспоненциального преобразования Фурье с использованием метода собственных значений получены аналитические решения. Выполнены численные расчеты для среды со свойствами меди в случае низкой, нормальной и высокой проводимости. Показано, что параметр дробного порядка оказывает существенное влияние на расчет физических величин в задаче.

Ключевые слова: метод собственных значений, модель Грина-Нагди типа III, теория обобщенной термоупругости с производной дробного порядка
\end{abstract}

\section{Introduction}

Many existing models of physical processes have been modified successfully by using the fractional calculus. A series of integral theories and fractional derivatives was created in the last half of the last century. Various approaches and definitions of fractional derivatives have become the main object of numerous studies [1,2]. Recently, to investigate the anomalous diffusion, a considerable research effort has been expended, which is characterized by the frac- tional time equation of wave diffusion by Kimmich [3] as in the form below

$$
\rho c=k I^{\alpha} c_{, i i}, i=1,2,3,
$$

where $k$ is the diffusion conductivity, $\rho$ is the mass density, $c$ is the concentration and $I^{\alpha}$ is the fraction of RiemannLiouville integral operator of order $\alpha$. It introduced as a natural generalization of the well-known integral $I^{\alpha} f(t)$ repeated $m$ times and wrote in the form of convolution type [4] 


$$
I^{\alpha} f(t)=\left\{\begin{array}{l}
\frac{1}{\Gamma(\alpha)} \int_{0}^{t}(t-\xi)^{\alpha} f(\xi) \mathrm{d} \xi, 0<\alpha \leq 2, \\
f(t), \alpha=0,
\end{array}\right.
$$

where $\Gamma(\alpha)$ is the Gamma function. The fractional order of weak, normal and strong heat conductivity under generalized thermoelastic theory was applied by Youssef [5] in the following form

$$
q_{i}+\tau_{0} \frac{\partial q_{i}}{\partial t}=-K I^{\alpha-1} \nabla T, 0<\alpha<2 .
$$

By using Taylor expansion of time-fractional order, Ezzat and El-Karamany [6] proposed a new fractional order generalized thermoelasticity model, which developed by Jumarie [7] as

$$
q_{i}+\frac{\tau_{0}^{\alpha}}{\Gamma(\alpha+1)} \frac{\partial^{\alpha} q_{i}}{\partial t^{\alpha}}=-K \nabla T, 0<\alpha \leq 1 .
$$

The fractional order study of generalized thermoelastic problems is an important branch in solid mechanics [812]. In addition, Abbas [13] studied the effects of fractional order and magnetic field in a thermoelastic medium due to moving heat source using the eigenvalue approach. Sherief and Abd El-Latief [14] studied the effect of the fractional order parameter and the variable thermal conductivity on a thermoelastic half-space. Due to thermal source, the effect of fractional order parameter on plane deformation in a thermoelastic medium was studied by Kumar et al. [15]. Abbas and Youssef [16] studied a two-dimensional thermoelastic porous material under fractional order theory. The fractional order influence in a functional graded thermoelastic material problem has been solved by Abbas [17]. Youssef and Abbas [18] studied the theory of generalized thermoelasticity with fractional order derivative in the case of variable thermal conductivity. Based upon the theory of two-temperature generalized thermoelasticity, Zenkour and Abouelregal [19] investigated the fractional heat conduction for an unbounded medium with a spherical cavity. Abbas [20] studied the solution of thermoelastic diffusion problem under fractional order theory in an infinite elastic medium with a spherical cavity.

In this work, the eigenvalue approach has been used to obtain the analytical solutions for temperature, displacement and the stress components. By employing an analytical-numerical technique based on the eigenvalues approach with Laplace and Fourier transformations, the nondimensional equations have been handled. Numerical computations for copper-like medium have been done for strong, normal and weak conductivity and the effect of the fractional order parameter has been estimated.

\section{Basic equations}

Let us consider a homogeneous, thermoelastic isotropic half-space $y \geq 0$ at initial uniform temperature $T_{0}$. Cartesian coordinate system $(x, y, z)$ has been used with $y$ axis is taken perpendicular to the bounding plane (Fig. 1). The displacement vector has the form $\mathbf{u}=(u, v, 0)$. The governing equations have the following form

$$
\begin{aligned}
& (\lambda+2 \mu) \frac{\partial^{2} u}{\partial x^{2}}+(\lambda+\mu) \frac{\partial^{2} v}{\partial x \partial y}+\mu \frac{\partial^{2} u}{\partial y^{2}}-\gamma \frac{\partial T}{\partial x}=\rho \frac{\partial^{2} u}{\partial t^{2}}, \\
& (\lambda+2 \mu) \frac{\partial^{2} v}{\partial y^{2}}+(\lambda+\mu) \frac{\partial^{2} u}{\partial x \partial y}+\mu \frac{\partial^{2} v}{\partial x^{2}}- \\
& -\gamma \frac{\partial T}{\partial y}=\rho \frac{\partial^{2} v}{\partial t^{2}}, \\
& I^{\alpha-1}\left(K^{*}+K \frac{\partial}{\partial t}\right)\left(\frac{\partial^{2} T}{\partial x^{2}}+\frac{\partial^{2} T}{\partial y^{2}}\right)= \\
& =\frac{\partial^{2}}{\partial t^{2}}\left(\rho c_{\mathrm{e}} T+\gamma T_{0}\left(\frac{\partial u}{\partial x}+\frac{\partial v}{\partial y}\right)\right),
\end{aligned}
$$

where the operator of fractional integral can be defined as the following [21]:

$$
\begin{aligned}
& I^{\alpha} f(\varphi)=\frac{1}{\Gamma(\alpha)} \int_{0}^{\varphi}(\varphi-\varepsilon)^{\alpha-1} f(\varepsilon) \mathrm{d} \varepsilon, \\
& \left\{\begin{array}{l}
0<\alpha<1 \text { for weak conductivity, } \\
\alpha=1 \text { for normal conductivity, } \\
1<\alpha \leq 2 \text { for strong conductivity, }
\end{array}\right. \\
& \sigma_{x x}=(\lambda+2 \mu) \frac{\partial u}{\partial x}+\lambda \frac{\partial v}{\partial y}-\gamma\left(T-T_{0}\right), \\
& \sigma_{y y}=(\lambda+2 \mu) \frac{\partial v}{\partial y}+\lambda \frac{\partial u}{\partial x}-\gamma\left(T-T_{0}\right), \\
& \sigma_{x y}=\mu\left(\frac{\partial u}{\partial x}+\frac{\partial v}{\partial y}\right),
\end{aligned}
$$

where $\lambda$ and $\mu$ are the elastic parameters, $T$ is the increment of temperature, $\rho$ is the density of mass, $\sigma_{x x}, \sigma_{x y}$ and $\sigma_{y y}$ are the stress components, $T_{0}$ is the body reference temperature, $c_{\mathrm{e}}$ is the specific heat at constant strain, $K$ is the thermal conductivity, $\gamma=(2 \lambda+3 \mu) \alpha_{t}$, and $\alpha_{t}$ is the linear thermal expansion coefficient. For convenience, the nondimensional variables can be introduced on the following form:

$$
\begin{aligned}
& \left(u^{\prime}, v, x^{\prime}, y^{\prime}\right)=\frac{c}{\varsigma}(u, v, x, y), T^{\prime}=\frac{T-T_{0}}{T_{0}}, \\
& \left(\sigma_{x x}^{\prime}, \sigma_{y y}^{\prime}, \sigma_{x y}^{\prime}\right)=\frac{1}{\mu}\left(\sigma_{x x}, \sigma_{y y}, \sigma_{x y}\right), t=\frac{c^{2}}{\varsigma} t,
\end{aligned}
$$

where $c^{2}=(\lambda+2 \mu) / \rho, \varsigma=K /\left(\rho c_{\mathrm{e}}\right)$.

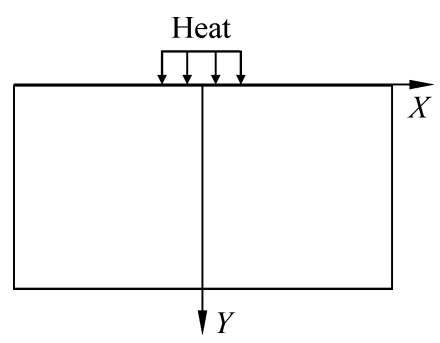

Fig. 1. Geometry of the problem 
In terms of these nondimensional variables (12), Eqs. (5)-(11), after suppressing the primes, can be written as

$$
\begin{aligned}
& \beta \frac{\partial^{2} u}{\partial x^{2}}+(\beta-1) \frac{\partial^{2} v}{\partial x \partial y}+\frac{\partial^{2} u}{\partial x^{2}}-\varpi \frac{\partial T}{\partial x}=\beta \frac{\partial^{2} u}{\partial t^{2}}, \\
& \beta \frac{\partial^{2} v}{\partial y^{2}}+(\beta-1) \frac{\partial^{2} u}{\partial x \partial y}+\frac{\partial^{2} v}{\partial x^{2}}-\varpi \frac{\partial T}{\partial y}=\beta \frac{\partial^{2} v}{\partial t^{2}}, \\
& I^{\alpha-1}\left(\varepsilon_{1}+\frac{\partial}{\partial t}\right)\left(\frac{\partial^{2} T}{\partial x^{2}}+\frac{\partial^{2} T}{\partial y^{2}}\right)= \\
& =\left(\frac{\partial}{\partial t}+\tau_{0} \frac{\partial^{2}}{\partial t^{2}}\right)\left(T+\varepsilon_{2}\left(\frac{\partial u}{\partial x}+\frac{\partial v}{\partial y}\right)\right), \\
& \sigma_{x x}=\beta \frac{\partial u}{\partial x}+(\beta-2) \frac{\partial v}{\partial y}+\frac{\partial^{2} u}{\partial x^{2}}-\varpi T, \\
& \sigma_{y y}=\beta \frac{\partial v}{\partial y}+(\beta-2) \frac{\partial u}{\partial x}-\varpi T, \\
& \sigma_{x y}=\frac{\partial u}{\partial y}+\frac{\partial v}{\partial x},
\end{aligned}
$$

where $\beta=(\lambda+2 \mu) / \mu, \quad \varpi=\gamma T_{0} / \mu, \varepsilon_{1}=K^{*} /\left(\rho c^{2} c_{\mathrm{e}}\right)$, and $\varepsilon_{2}=\gamma /\left(\rho c_{\mathrm{e}}\right)$.

\section{Application}

Now, we can assume the homogeneous initial conditions as

$$
\begin{aligned}
& T(x, y, 0)=\frac{\partial T(x, y, 0)}{\partial t}=0 \\
& u(x, y, 0)=\frac{\partial u(x, y, 0)}{\partial t}=0 \\
& v(x, y, 0)=\frac{\partial v(x, y, 0)}{\partial t}=0 .
\end{aligned}
$$

The boundary conditions at $y=0$ for the present problem are supposed as

$$
T(x, 0, t)=T_{1} H(\alpha-|x|) H(t),
$$

where $T_{1}$ is a constant, $H$ is the unit step function (Heaviside function). It is means that the thermal shock acts on a band of a width $2 \alpha$ centered around the $x$ axis on the half-space surface $(y=0)$ and is zero elsewhere. On the boundary $y=0$, we suppose that the body displacement $v$ does not depend on $y$, which leads to

$$
\frac{\partial v(x, 0, t)}{\partial y}=0 \text {. }
$$

Moreover, the medium is subjected to rigid foundation and rough enough to prevent the displacement $u$ at any point of $x$ and any time $t$, which leads to

$$
u(x, 0, t)=0 \text {. }
$$

\section{Laplace and Fourier transformations}

Let us define the Laplace transformation for a function $\Omega(x, y, t)$ by

$$
\begin{aligned}
& L[\Omega(x, y, t)]=\bar{\Omega}(x, y, s)=\int_{0}^{\infty} \Omega(x, y, t) e^{-s t} \mathrm{~d} t, \\
& s>0,
\end{aligned}
$$

where $s$ is a parameter. Hence, the above equations will take the form:

$$
\begin{aligned}
& \beta \frac{\partial^{2} \bar{u}}{\partial x^{2}}+(\beta-1) \frac{\partial^{2} \bar{v}}{\partial x \partial y}+\frac{\partial^{2} \bar{u}}{\partial y^{2}}-\varpi \frac{\partial T}{\partial x}=\beta s^{2} \bar{u}, \\
& \beta \frac{\partial^{2} \bar{v}}{\partial y^{2}}+(\beta-1) \frac{\partial^{2} \bar{u}}{\partial x \partial y}+\frac{\partial^{2} \bar{v}}{\partial x^{2}}-\varpi \frac{\partial T}{\partial y}=\beta s^{2} \bar{v} \\
& \frac{\partial^{2} \bar{T}}{\partial x^{2}}+\frac{\partial^{2} \bar{T}}{\partial y^{2}}=\frac{s^{\alpha-1}}{\varepsilon_{1}+s}\left(\bar{T}+\varepsilon_{2}\left(\frac{\partial \bar{u}}{\partial x}+\frac{\partial \bar{v}}{\partial y}\right)\right), \\
& \bar{\sigma}_{x x}=\beta \frac{\partial \bar{u}}{\partial x}+(\beta-2) \frac{\partial \bar{v}}{\partial y}-\varpi \bar{T}, \\
& \bar{\sigma}_{y y}=\beta \frac{\partial \bar{v}}{\partial y}+(\beta-2) \frac{\partial \bar{u}}{\partial x}-\varpi \bar{T} \\
& \bar{\sigma}_{x y}=\frac{\partial \bar{u}}{\partial y}+\frac{\partial \bar{v}}{\partial x}, \\
& \bar{T}(x, 0, s)=\frac{T_{1} H(\alpha-|x|)}{s} \\
& \frac{\partial \bar{v}(x, 0, s)}{\partial y}=0, \bar{u}(x, 0, s)=0 .
\end{aligned}
$$

Now, the exponential Fourier transformation for the function $\bar{\Omega}(x, y, s)$ can be given by

$$
\bar{\Omega}^{*}(q, y, s)=\int_{-\infty}^{\infty} \bar{\Omega}(x, y, s) e^{-i q x} \mathrm{~d} x .
$$

Hence, the above differential equations can assume the form:

$$
\begin{aligned}
& -q^{2} \beta \bar{u}^{*}+(\beta-1) i q \frac{\mathrm{d} \bar{v}^{*}}{\mathrm{~d} y}+\frac{\mathrm{d}^{2} \bar{u}^{*}}{\mathrm{~d} y^{2}}-i q \varpi \bar{T}^{*}=\beta s^{2} \bar{u}^{*}, \\
& \beta \frac{\mathrm{d}^{2} \bar{v}^{*}}{\mathrm{~d} y^{2}}+i q(\beta-1) \frac{\mathrm{d} \bar{u}^{*}}{\mathrm{~d} y}+q^{2} \bar{v}^{*}-\varpi \frac{\mathrm{d} T^{*}}{\mathrm{~d} y}=\beta s^{2} \bar{v}^{*}, \\
& -q^{2} \bar{T}^{*}+\frac{\mathrm{d}^{2} T}{\mathrm{~d} y^{2}}=\frac{s^{\alpha+1}}{\varepsilon_{1}+s}\left(\bar{T}^{*}+\varepsilon_{2}\left(i q \bar{u}^{*}+\frac{\mathrm{d} \bar{v}^{*}}{\mathrm{~d} y}\right)\right), \\
& \bar{\sigma}_{x x}^{*}=i q \beta \bar{u}^{*}+(\beta-2) \frac{\mathrm{d} \bar{v}^{*}}{\mathrm{~d} y}-\varpi \bar{T}^{*}, \\
& \bar{\sigma}_{y y}^{*}=\beta \frac{\mathrm{d} \bar{v}^{*}}{\mathrm{~d} y}+i q(\beta-2) \bar{u}^{*}-\varpi \bar{T}^{*}, \\
& \bar{\sigma}_{x y}^{*}=\frac{\mathrm{d} \bar{u}^{*}}{\mathrm{~d} y}+i q \bar{v}^{*}, \\
& \frac{\mathrm{d} \bar{v}^{*}(q, 0, s)=T \sqrt{\frac{2}{\pi}} \frac{\sin (q \alpha)}{q s},}{\mathrm{~d} y}=0, \bar{u}^{*}(q, 0, s)=0 .
\end{aligned}
$$

By using the eigenvalue approach as made in Refs. [22, 23 ], let us now proceed to solve the coupled differential equations (32)-(34). In the form of vector-matrix differential equation, Eqs. (32)-(34) can be written as follows 


$$
\frac{\mathrm{d} \vartheta}{\mathrm{d} y}=\mathbf{A} \vartheta
$$

where

$$
\vartheta=\left[\bar{v}^{*} \bar{u}^{*} \bar{T}^{*} \frac{\mathrm{d} \bar{v}^{*}}{\mathrm{~d} y} \frac{\mathrm{d} \bar{u}^{*}}{\mathrm{~d} y} \frac{\mathrm{d} \bar{T}^{*}}{\mathrm{~d} y}\right]^{\mathrm{T}}
$$

and

$$
\mathbf{A}=\left[\begin{array}{cccccc}
0 & 0 & 0 & 1 & 0 & 0 \\
0 & 0 & 0 & 0 & 1 & 0 \\
0 & 0 & 0 & 0 & 0 & 1 \\
a_{41} & 0 & 0 & 0 & a_{45} & a_{46} \\
0 & a_{52} & a_{53} & a_{54} & 0 & 0 \\
0 & a_{62} & a_{63} & a_{64} & 0 & 0
\end{array}\right] .
$$

Thus, the matrix $\mathbf{A}$ has the characteristic equation in the form

$$
\xi^{6}-M_{1} \xi^{4}+M_{2} \xi^{2}+M_{3}=0,
$$

where

$$
\begin{aligned}
& M_{1}=a_{41}+a_{52}+a_{45} a_{54}+a_{63}+a_{46}, \\
& M_{2}=a_{41} a_{52}-a_{53} a_{62}-a_{46} a_{54} a_{62}+a_{41} a_{63}+ \\
& \quad+a_{52} a_{63}+a_{45} a_{54} a_{63}+a_{46} a_{52} a_{64}-a_{45} a_{53} a_{64}, \\
& M_{3}=a_{41} a_{53} a_{62}-a_{41} a_{52} a_{63} .
\end{aligned}
$$

The eigenvalues of matrix $\mathbf{A}$ are the roots of Eq. (40) in the

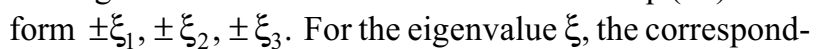
ing eigenvector $\mathbf{Y}=\left[Y_{1}, Y_{2}, Y_{3}, Y_{4}, Y_{5}, Y_{6}\right]$ can be calculated as:

$$
\begin{aligned}
& Y_{1}=-a_{45} a_{54} \xi+a_{46} \xi\left(a_{52}-\xi^{2}\right), \\
& Y_{2}=-a_{46} a_{54} \xi^{2}+a_{53}\left(a_{41}-\xi^{2}\right), \\
& Y_{3}=-a_{41} a_{52}+\left(a_{41}+a_{52}+a_{45} a_{54}\right) \xi^{2}-\xi^{4}, \\
& Y_{4}=\xi Y_{4}, Y_{5}=\xi Y_{2}, Y_{6}=\xi Y_{3} .
\end{aligned}
$$

For further reference, the following notations can be used:

$$
\begin{aligned}
& Y_{1}=[\mathbf{Y}]_{\xi=-\xi_{1}}, Y_{2}=[\mathbf{Y}]_{\xi=-\xi_{2}}, Y_{3}=[\mathbf{Y}]_{\xi=-\xi_{3}}, \\
& Y_{4}=[\mathbf{Y}]_{\xi=\xi_{1}}, Y_{5}=[\mathbf{Y}]_{\xi=\xi_{2}}, Y_{6}=[\mathbf{Y}]_{\xi=\xi_{3}} .
\end{aligned}
$$

The solution of Eq. (39) can be given in the following form:

$$
\vartheta(q, y, s)=\sum_{i=1}^{3} B_{i} Y_{i} e^{-\xi_{i} y}
$$

Due to the regularity conditions of the solution, the exponential in the space variable $x$ has been discarded at infinity. By using the problem boundary conditions, the constants $B_{1}, B_{2}$ and $B_{3}$ have been calculated.

\section{Double transformation inversion}

The expression $\bar{\Omega}^{*}(q, y, s)$ in the domain of Fourier transformation can be expressed as

$$
\overline{\bar{\Omega}}(q, y, s)=\frac{1}{\sqrt{2 \pi}} \int_{-\infty}^{\infty} \bar{\Omega}^{*}(q, y, s) e^{i q x} \mathrm{~d} q .
$$

Thus, the physical quantities can be given for $x, y$ and $s$ in the forms:

$$
\bar{u}(x, y, s)=\frac{1}{\sqrt{2 \pi}} \sum_{k=1}^{3} \int_{-\infty}^{\infty} B_{k} u_{k} e^{-\xi_{k} y+i q x} \mathrm{~d} q,
$$

$$
\begin{aligned}
& \bar{v}(x, y, s)=\frac{1}{\sqrt{2 \pi}} \sum_{k=1}^{3} \int_{-\infty}^{\infty} B_{k} v_{k} e^{-\xi_{k} y+i q x} \mathrm{~d} q, \\
& \bar{T}(x, y, s)=\frac{1}{\sqrt{2 \pi}} \sum_{k=1}^{3} \int_{-\infty}^{\infty} B_{k} T_{k} e^{-\xi_{k} y+i q x} \mathrm{~d} q, \\
& \bar{\sigma}_{x x}(x, y, s)=\frac{1}{\sqrt{2 \pi}} \sum_{k=1}^{3} \int_{-\infty}^{\infty} B_{k}\left(i q \eta u_{k}-\right. \\
& \left.-\xi_{k}(\eta-2) v_{k}-\varsigma T_{k}\right) e^{-\xi_{k} y+i q x} \mathrm{~d} q, \\
& \bar{\sigma}_{y y}(x, y, s)=\frac{1}{\sqrt{2 \pi}} \sum_{k=1}^{3} \int_{-\infty}^{\infty} B_{k}\left(-\xi_{k} \eta v_{k}-\right. \\
& \left.\quad-i q(\eta-2) u_{k}-\varsigma T_{k}\right) e^{-\xi_{k} y+i q x} \mathrm{~d} q, \\
& \bar{\sigma}_{x y}(x, y, s)=\frac{1}{\sqrt{2 \pi}} \sum_{k=1}^{3} \int_{-\infty}^{\infty} B_{k}\left(-\xi_{k} u_{k}+\right. \\
& \left.\quad+i q v_{k}\right) e^{-\xi_{k} y+i q x} \mathrm{~d} q,
\end{aligned}
$$
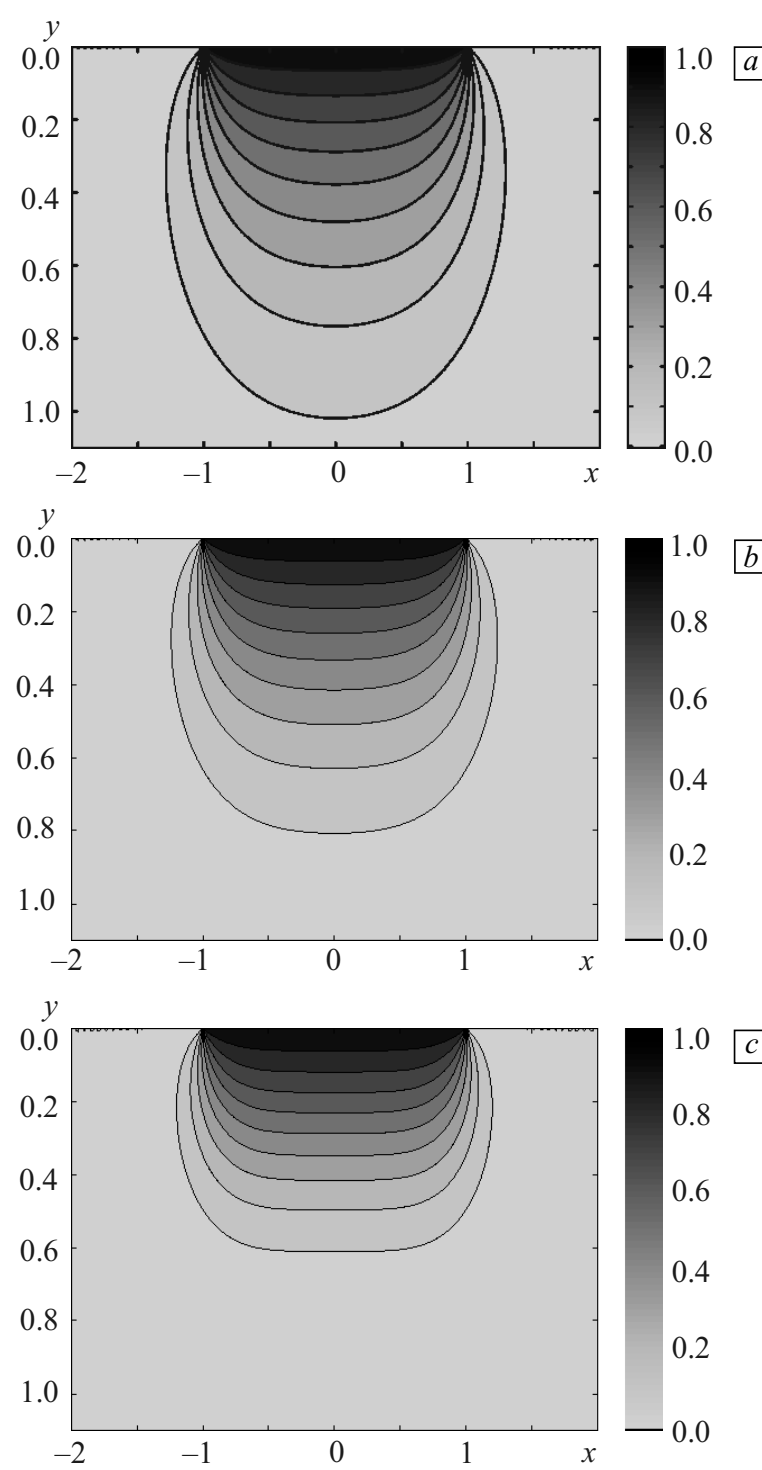

Fig. 2. Contour plots of the temperature distribution on the material for weak $(a)$, normal $(b)$ and strong conductivity $(c)$ at $t=0.12$ 
where $u_{k}, v_{k}$ and $T_{k}$ are the components of the corresponding eigenvectors. We adopt a numerical inversion method based on the Stehfest algorithm [24]. In this method, the inverse $g(x, y, t)$ of the Laplace transformation $\bar{g}(x, y, s)$ is approximated by the relation

$$
\Omega(x, y, t)=\frac{\ln 2}{t} \sum_{j=1}^{N} V_{j} \bar{\Omega}\left(x, y, \frac{\ln 2}{t} j\right),
$$

and $V_{j}$ takes the form

$$
\begin{aligned}
V_{j} & =(-1)^{(N / 2+1)} \times \\
\times & \sum_{k=(i+1) / 2}^{\min (i, N / 2)} \frac{k^{(N / 2+1)}(2 k) !}{(N / 2-k) ! k !(i-k) !(2 k-1) !}
\end{aligned}
$$

where $N$ is the term number used in summation in Eq. (52) and should be optimized by trial and error.

\section{Numerical results and discussion}

In order to illustrate the theoretical results obtained in the previous section, we present some numerical values for the physical constants. We assume that the plate is made of an isotropic material (copper). The physical constants are listed below [25]:
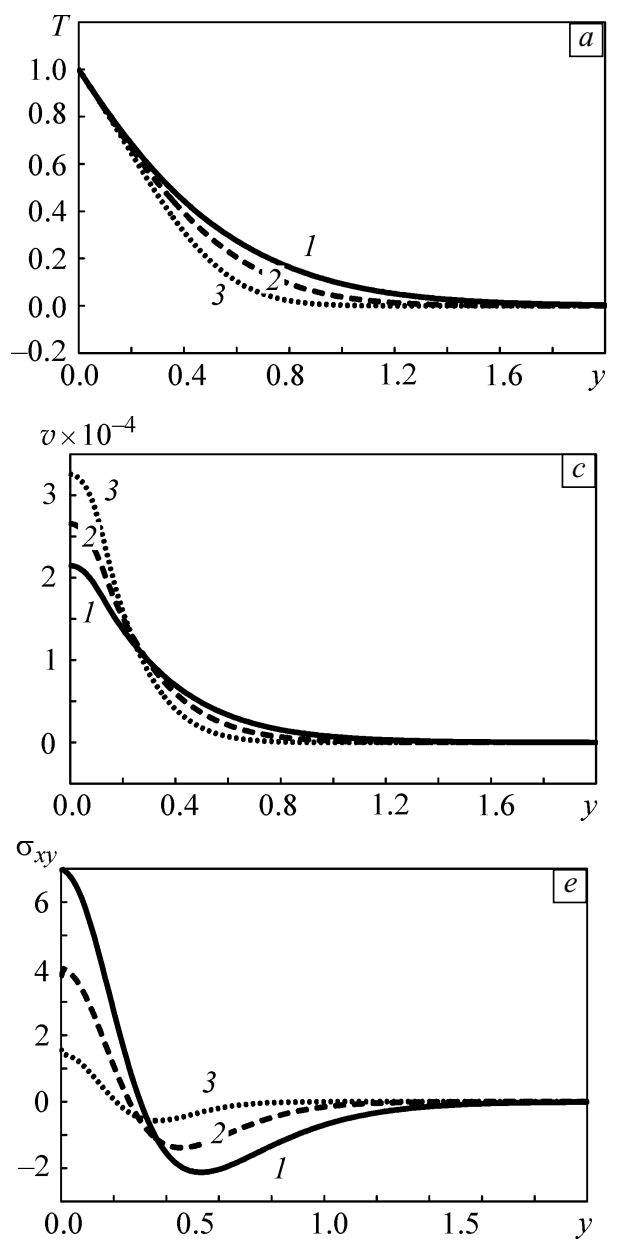

$$
\begin{aligned}
& \rho=8954 \mathrm{~kg} \mathrm{~m}^{-3}, \lambda=7.76 \times 10^{10} \mathrm{~N} \mathrm{~m}^{-2}, \\
& \mu=3.86 \times 10^{10} \mathrm{~N} \mathrm{~m}^{-2}, \alpha_{\mathrm{t}}=1.78 \times 10^{-5} \mathrm{~K}^{-1}, \\
& T_{0}=293 \mathrm{~K}, c_{\mathrm{e}}=383.1 \mathrm{~J} \mathrm{~kg}^{-1} \mathrm{~K}^{-1} .
\end{aligned}
$$

Here the graphs are plotted to display the variations of temperature, displacements and stresses for $T_{1}=1, \alpha=1$ at $t=0.12$. The results of the numerical evaluation of temperature distribution contours for weak, normal and strong conductivity are illustrated in Fig. 2. We can observed that the change of temperature area is limited within a finite area and the temperature does not change outside that area. In addition, we can find that the heat influence zone with weak conductivity gets greater than the heat influence area with normal conductivity which it is greater than the heat influence area with strong conductivity. Also, there are areas with a greater slope of the temperature of another zone. This means that heat conducts at a finite speed.

Figure 3 shows the variation of the nondimensional temperature, displacement components and stress components along the distance $y$ for different fractional order parameter values $\alpha=0.8,1.0$ and 1.2 at fixed time $(t=0.12)$ when $x$ direction $(x=0.5)$ remains constant. The solid
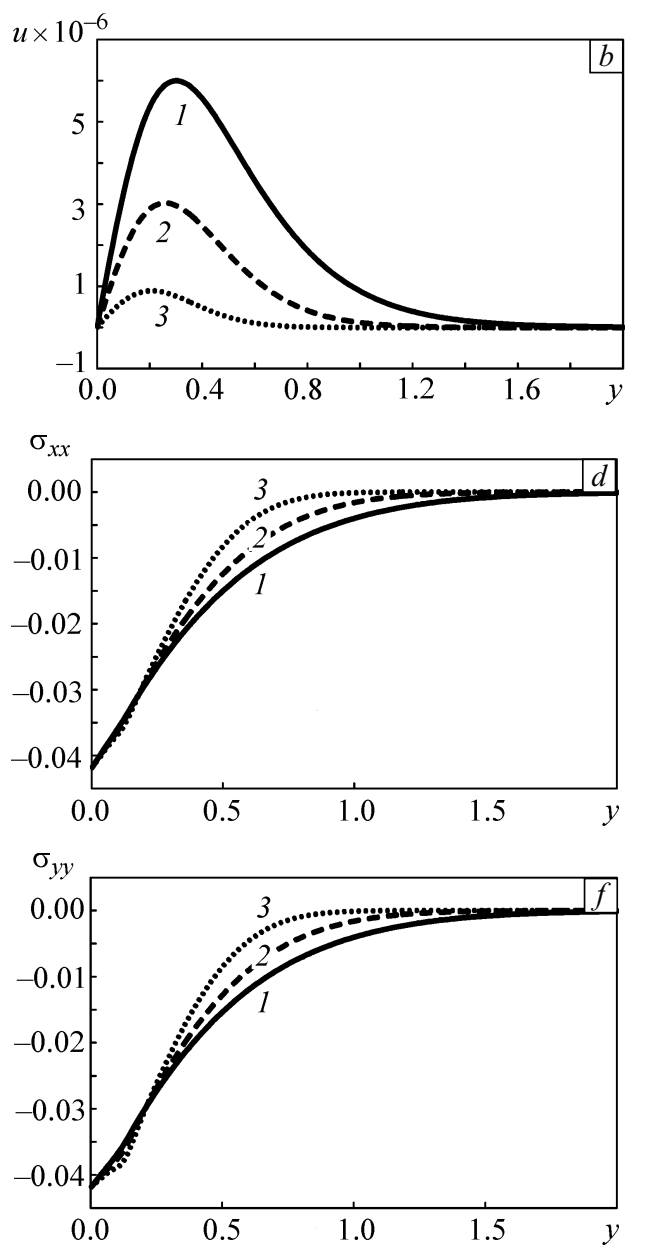

Fig. 3. The distribution of temperature $T(a)$, horizontal $u(b)$, vertical displacement $v(c), \sigma_{x x}(d), \sigma_{x y}(e)$, and $\sigma_{y y}(f)$ stress components with respect to $y$ for different values of $\alpha=0.8(1), 1.0(2), 1.2$ (3) when $x=0.5$ at $t=0.12$ 

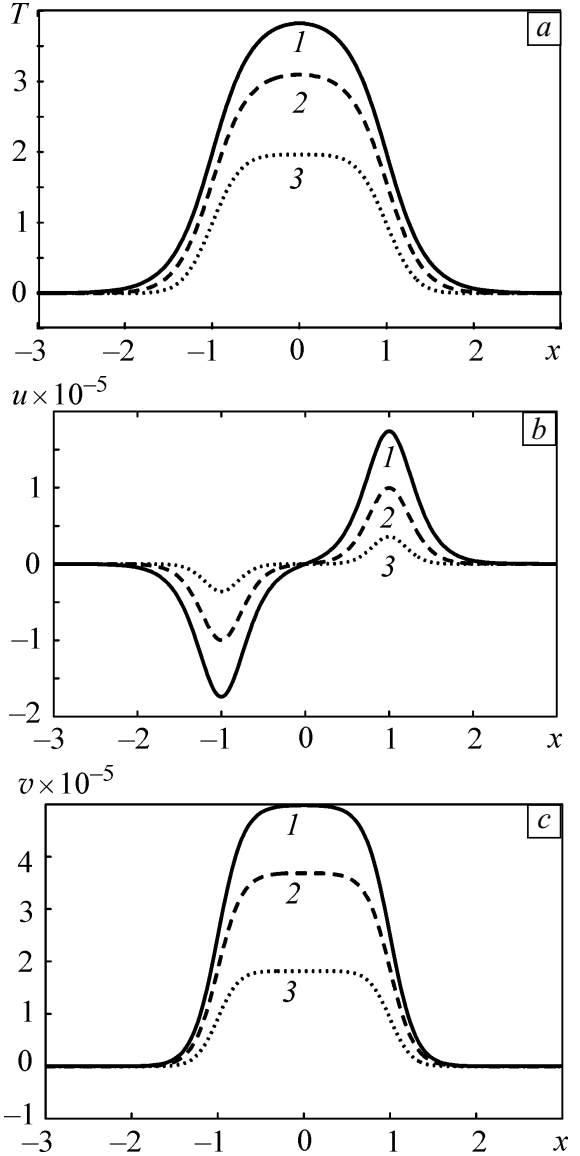

Fig. 4 The distribution of temperature $T(a)$, horizontal $u(b)$, vertical displacement $v(c)$ with respect to $x$ for different values of $\alpha=0.8$ (1), 1.0 (2), 1.2 (3) when $y=0.5$ at $t=0.12$

line (1) refers to the weak conductivity, while the dotted line (3) refers to the normal conductivity and the dashed line (2) refers to the strong conductivity. Figure 3, a represents the variation of temperature with the distance $y$. It is observed that at the boundary $y=0$, the magnitude of temperature is equal to one, which agrees with the imposed boundary condition. The magnitude of temperature starts from the maximum value and then continuously decreases to zero with increasing $y$, while it increases with decreasing the fractional parameter $\alpha$. Figure $3, b$ represents the variation of the horizontal displacement with the distance $y$. The horizontal displacement starts from zero which obey the boundary condition, then gradually increases until it attains a peak value at a particular location after that gradually decreases to zero with increasing $y$. The horizontal displacement increases with decreasing the fractional parameter. Figure 3, $c$ shows the variation of the vertical displacement with the distance $y$. The magnitude of vertical displacement starts from the maximum value and then continuously decreases to close zero with increasing $y$. Also, before the intersection of the three curves, the vertical displacement magnitude decreases as the value of the fractional order decreases.
Figures 3, $d-f$ depict stress components $\sigma_{x x}, \sigma_{x y}$ and $\sigma_{y y}$ with respect to the distance $y$. The magnitude of stress components $\sigma_{x x}, \sigma_{x y}$ and $\sigma_{y y}$ decreases as the value of the fractional order decreases before the intersection of the three curves. However, after the intersection, all values increase as the fractional order decreases.

Figure 4 demonstrate the variation of the nondimensional forms of temperature and the displacement components with the distance $x$ for different fractional order parameter values $\alpha=0.8,1.0$ and 1.2 at fixed time $(t=0.12)$ when the $y$ direction $(y=0.5)$ remains constant. The solid line (1) refers to the weak conductivity, while the dotted line (3) refers to the normal conductivity and the dashed line (2) refers to the strong conductivity.

In Fig. 4, $a$ the temperature distribution is plotted along the distance $x$ for $y=0.5$. At the length of the surface heated $(-1 \leq x \leq 1)$, the temperature field has maximum values and begins to decrease near the edges $(x= \pm 1)$ where it decreases smoothly and finally closes zero. Figure $4, b$ displays the distribution of the horizontal displacement along the distance $x$. The vertical displacement starts decreasing at the beginning and ending of the surface heated $(-1 \leq x \leq$ $\leq 1)$ and has a minimum value at the middle of the heated surface, after which it begins to increase and reaches a maximum close to the edges $(x= \pm 1)$ and then decreases to zero. The variation of the vertical displacement with respect to $x$ is shown in Fig. 4, $c$. The displacement magnitude has the maximum value along the length of the surface heated $(-1 \leq x \leq 1)$, and it starts to decrease just near the edges $(x= \pm 1)$, and then decreases to zero.

\section{Conclusions}

The effect of fractional order derivative on a two-dimensional problem due to thermal shock with weak, normal and strong conductivity has been investigated. The governing equations have been taken in the context of Green and Naghdi of type III model (GNIII model) under fractional order derivative. An analytical solutions has been obtained by employing the Laplace and exponential Fourier transformations in combination with the eigenvalues approach. For weak, normal and strong conductivity, some numerical computations for a copper-like medium have been carried out. The results demonstrate that the effect of fractional order parameter has a significant role for all physical quantities considered in the problem.

\section{References}

1. Oldham K.B., Spanier J. The Fractional Calculus. V. 111 // Mathematics in Science and Engineering. - New York-London: Academic Press, 1974.

2. Podlubny I. Fractional Differential Equations: An Introduction to Fractional Derivatives, Fractional Differential Equations, to Methods of Their Solution and Some of Their Applications. V. 198 // Mathematics in Science and Engineering. - San Diego: Academic Press, 1999.

3. Kimmich R. Strange kinetics, porous media, and NMR// Chem. Phys. 2002. - V. 284. - No. 1-2. - P. 253-285. 
4. Mainardi F., Gorenflo R. On Mittag-Leffler-type functions in fractional evolution processes // J. Comput. Appl. Math. -2000. - V. 118. No. 1. - P. 283-299.

5. Youssef H.M. Theory of fractional order generalized thermoelasticity // J. Heat Trans. - 2010. - V. 132. - No. 6. - P. 1-7.

6. Ezzat M.A., El Karamany A.S. Theory of fractional order in electrothermoelasticity // Eur. J. Mech. A. Solid. - 2011. - V. 30. - No. 4. P. 491-500.

7. Jumarie G. Derivation and solutions of some fractional black-scholes equations in coarse-grained space and time // Appl. Merton's Opt. Portfolio. Comput. Math. Appl. - 2010. - V. 59. - No. 3. - P. 11421164.

8. Mashat D.S., Zenkour A.M., Abouelregal A.E. Fractional order thermoelasticity theory for a half-space subjected to an axisymmetric heat distribution // Mech. Adv. Mater. Struct. - 2015. - V. 22. - No. 11. P. 925-932.

9. Sur A., Kanoria M. Fractional order generalized thermoelastic functionally graded solid with variable material properties // J. Solid Mech. - 2014. - V. 6. - No. 1. - P. 54-69.

10. Sarkar N., Lahiri A. The effect of fractional parameter on a perfect conducting elastic half-space in generalized magneto-thermoelasticity // Meccanica. - 2013. - V. 48. - No. 1. - P. 231-245.

11. Youssef H.M. Two-dimensional thermal shock problem of fractional order generalized thermoelasticity // Acta Mech. - 2012. - V. 223. No. 6. - P. 1219-1231.

12. Ezzat M.A., El-Karamany A.S., Fayik M.A. Fractional ultrafast laserinduced thermo-elastic behavior in metal films // J. Thermal Stress. 2012. - V. 35. - No. 7. - P. 637-651.

13. Abbas I.A. Eigenvalue approach to fractional order generalized magneto-thermoelastic medium subjected to moving heat source // J. Magnetism Magnet. Mater. - 2015. - V. 377. - P. 452-459.

14. Sherief H., Abd El-Latief A.M. Effect of variable thermal conductivity on a half-space under the fractional order theory of thermoelasticity // Int. J. Mech. Sci. - 2013. - V. 74. - P. 185-189.
15. Kumar R., Gupta V., Abbas I.A. Plane deformation due to thermal source in fractional order thermoelastic media // J. Comput. Theor. Nanosci. - 2013. - V. 10. - No. 10. - P. 2520-2525.

16. Abbas I.A., Youssef H.M. Two-dimensional fractional order generalized thermoelastic porous material // Latin Am. J. Solid. Struct. 2015. - V. 12. - No. 7. - P. 1415-1431.

17. Abbas I.A. A Problem on functional graded material under fractional order theory of thermoelasticity // Theor. Appl. Fract. Mech. - 2014. V. 74. - P. $18-22$.

18. Youssef H.M., Abbas I.A. Fractional order generalized thermoelasticity with variable thermal conductivity // J. Vibroeng. - 2014. - V. 16. No. 8. - P. 4077-4087.

19. Zenkour A.M., Abouelregal A.E. State-space approach for an infinite medium with a spherical cavity based upon two-temperature generalized thermoelasticity theory and fractional heat conduction // Zeitsch. Angewandte Math. Phys. - 2014. - V. 65. - No. 1. - P. 149-164.

20. Abbas I.A. Eigenvalue approach for an unbounded medium with a spherical cavity based upon two-temperature generalized thermoelastic theory // J. Mech. Sci. Tech. - 2014. - V. 28. - No. 10. - P. 4193-4198.

21. Sur A., Kanoria M. Fractional heat conduction with finite wave speed in a thermo-visco-elastic spherical shell // Latin Am. J. Solid. Struct. 2014. - V. 11. - No. 7. - P. 1132-1162.

22. Das N.C., Lahiri A., Giri R.R. Eigenvalue approach to generalized thermoelasticity // Ind. J. Pure Appl. Math. - 1997. - V. 28. - No. 12. P. $1573-1594$.

23. Abbas I.A. A Dual phase lag model on thermoelastic interaction in an infinite fiber-reinforced anisotropic medium with a circular hole // Mech. Based Design Struct. Machin. - 2015. - V. 43. - No. 4. P. 501-513.

24. Stehfest $H$. Algorithm 368: Numerical inversion of laplace transforms [D5] // Commun. ACM. - 1970. - V. 13. - No. 1. - P. 47-49.

25. Sherief H.H., El-Maghraby N.M. A mode-I crack problem for an infinite space in generalized thermoelasticity // J. Therm. Stress. 2005. - V. 28. - No. 5. - P. 465-484. 